\title{
Strategies for management of diabetes in the young in China
}

\author{
Xiaoping Luo \\ From 8th APPES Biennial Scientific Meeting \\ Darwin, Australia. 29 October - 1 November 2014
}

The incidence of type 1 diabetes mellitus (T1DM) is rising rapidly in children, with an overall annual global increase of 3\%. Approximately 79,100 children under age 15 develop T1DM annually. Although the reported incidence of T1DM in China was as low as 0.6 per 100,000 per annum in 1995, the number of children with T1DM cannot be underestimated within the large population. The number of type 2 diabetics in the young has been increasing significantly in the last decades.

To improve access to quality care for children with diabetes in China, the World Diabetes Foundation (WDF) planned a project to help to establish pediatric diabetes centers to increase the rate of diagnosis and to improve the quality of treatment for children with diabetes through training of health care professionals. A survey was conducted on the basis of pediatric diabetes centers across China to raise the awareness of pediatric diabetes, and to evaluate the diagnosis and treatment status of diabetes care in children. Multiple diabetes camps were organized for children and caregivers. The project commenced in December 2012 and will be completed in February 2015. Thirty three pediatric diabetes centers in 25 cities across China were established. A cross-sectional questionnaire survey about the children with diabetes was completed.

Until the end of December 2013, 1,224 children with T1DM were effectively enrolled into the program with a mean age of $7.28 \pm 3.75$ (mean $\pm \mathrm{SD}$ ). For the age distribution, $79 \%$ were in the range of 4-12 years old, of those 384 cases were younger than 5 and 840 cases were older than 5 years old. The male to female ratio was $46.8 \%$ to $53.2 \%$. $49 \%$ of the patients presented with typical diabetic symptoms such as increased thirst, frequent urination, extreme hunger, weight loss and fatigue. 59.3\% cases first presented with diabetic ketoacidosis (DKA). The overall incidence of hypoglycemia was $17.5 \%$ (20.3\% in $<5 y$ vs $16.3 \%$ in $\geq 5 y$,
P $>0.05 y$ ). The total incidence of DKA was $59.3 \%$ (57.4\% in $<5 y$ vs $60.2 \%$ in $\geq 5 y, P>0.05)$. The average level of HbA1c was $(11.1 \pm 3.2) \%$. Using HbA1c $7.5 \%$ as a cut-off, the incidence of hypoglycemia was $29.3 \%$ in the HbA1c below $7.5 \%$ group and $14.2 \%$ in the above $7.5 \%$ group $(\mathrm{P}<0.001)$. The incidence of DKA was $54 \%$ in the HbA1c below $7.5 \%$ group and $61 \%$ in the above $7.5 \%$ group $(P>0.05) .97 .2 \%$ of the patients received insulin treatment, while $83.3 \%$ with insulin injection and $14 \%$ adopted pump therapy. $78.8 \%$ of the patients were using multiple insulin injection regimes.

In China, T1DM is still the major form of diabetes among the young, although there is a tendency of increasing T2DM. Greater efforts need to be made to improve the awareness and quality of care. A task fprce on diabetes in children was recently formed by the Chinese Society of Pediatric Endocrinology and Metabolism (CSMEM) to facilitate nationwide research and collaboration.

Published: 28 April 2015

doi:10.1186/1687-9856-2015-S1-017

Cite this article as: Luo: Strategies for management of diabetes in the young in China. International Journal of Pediatric Endocrinology 20152015 (Suppl 1):017.
Tongji Hospital, Wuhan, China

\section{() Biomed Central}

(c) 2015 Luo; licensee BioMed Central Ltd. This is an Open Access article distributed under the terms of the Creative Commons Attribution License (http://creativecommons.org/licenses/by/4.0), which permits unrestricted use, distribution, and reproduction in any medium, provided the original work is properly cited. The Creative Commons Public Domain Dedication waiver (http:// creativecommons.org/publicdomain/zero/1.0/) applies to the data made available in this article, unless otherwise stated. 